Hydroécol. Appl. (1990) 1/2, pp. 21-36

\title{
Statistiques de température de la Manche le long des côtes Nord Bretagne et Ouest Normandie : années 1962 à 1987
}

\author{
Ph. Gosse et J. Riou \\ Électricité de France - Direction des Études et Recherches \\ 6, quai Watier, 78400 Chatou - France
}

Résumé. - On donne les fréquences de dépassement des seuils thermiques 17, 18, 19 et $20^{\circ} \mathrm{C}$ à la côte entre Brest et Port-en-Bessin en exploitant deux modèles numériques qui calculent les températures journalières de la Manche à partir des coefficients de marée et du réseau météo d'observations atmosphériques: le premier modèle intéresse la bande côtière Roscoff - St Malo avec une résolution spatiale de $2 \times 2 \mathrm{~km}^{2}$ et une précision meilleure que $1^{\circ} \mathrm{C}$; le deuxième, un peu moins fiable dans le domaine étudié, a été développé sur l'ensemble de la Manche avec un pas d'espace de $11 \times 11 \mathrm{~km}^{2}$.

On met bien en évidence les deux endroits les plus chauds en été : le golfe normano-breton et l'intervalle pointe de Barfleur - Port-en-Bessin; en particulier, la température $18^{\circ} \mathrm{C}$ y est dépassée plus de 30 jours par an en moyenne, alors que cet événement survient moins de 1 jour par an dans le reste du domaine étudié.

Summary. - Two numerical models calculating the daily temperatures of the English Channel from tide coefficients and atmospheric meteorological observations are exploited in order to determine the frequencies of temperatures above $17,18,19$ and $20^{\circ} \mathrm{C}$ along the coast between Brest and Port-en-Bessin, The first model concerns the Roscoff-St Malo coastal strip, detailed with a $2 \times 2 \mathrm{~km}^{2}$ spatial resolution and an accuracy better than $1^{\circ} \mathrm{C}$. The second one, slightly less accurate in the area under study, describes the entire Channel with a $11 \times 11 \mathrm{~km}^{2}$ spatial resolution. The two warmest places are disclosed: the Norman-Breton bay and the Barfleur - Port-en-Bessin interval. In particular, in this area, the threshold $18^{\circ} \mathrm{C}$ is exceeded more than 30 days per year on average while this happens less than one day per year elsewhere. 


\section{1. - INTRODUCTION}

Si la recherche de fréquences de dépassement de températures élevées le long des côtes de la Manche peut présenter un intérêt certain pour des activités récréatives comme la baignade, elle est essentielle pour bien connaître la tolérance du milieu marin à des apports côtiers d'origine urbaine, industrielle ou agricole: les températures élevées accélèrent en effet beaucoup de cinétiques chimiques et biologiques et peuvent ainsi favoriser des réactions indésirables d'un écosystème marin enrichi par des apports côtiers, par exemple des proliférations d'espèces phytoplanctoniques dont certaines peuvent être toxiques.

Dans cette optique, nous mettons ici en évidence les zones côtières les plus chaudes en été en Bretagne Nord et Ouest Normandie, en examinant les fréquences de dépassement des températures $17,18,19$ et $20^{\circ} \mathrm{C}$.

Comple tenu de l'inexistence d'un réseau de mesures en continu des températures le long de la côte, le meilleur moyen pour les reconstituer consiste à utiliser des modèles numériques déterministes qui ont été appliqués avec succès en mer à EDF (Klein, 1980; Agoumi et al., 1982; Riou, 1989) dans la poursuite d'études entreprises sur des rivières (Gras, 1969; Poulin, 1980; Gras et al., 1986), puis des lacs (Klein et Bonnaud, 1977; Enderlé, 1980; Salençon, 1987) : le principe de ces modèles est de calculer avec un pas de temps de l'ordre de l'heure les températures d'un écoulement aquatique soumis à des échanges d'énergie à l'interface eau-atmosphère (ces échanges sont calculés à partir des observations atmosphériques trihoraires d'une station météorologique proche) et à des frictions sur le fond (le flux d'énergie turbulente ainsi généré sur la verticale est calculé à partir de la vitesse de l'écoulement).

Dans le cas de la Manche, on utilise les données météorologiques de la station représentative de La Hague, les tableaux de coefficients de marée, et les cartes de courants de marée calculés pour quelques coefficients par le Laboratoire National d'Hydraulique.

Deux modèles thermiques de la Manche sont utilisés dans cette étude; tous deux ont une structure bicouche sur la verticale, ils permettent de calculer des températures de surface et de fond: le premier appelé modèle global, développé sur l'ensemble de la Manche, calcule les évolutions thermiques avec un pas de temps trihoraire et un pas d'espace de $11 \times 11 \mathrm{~km}^{2}$ (figure 8); le deuxième appelé modèle côtier, d'emprise plus réduite, intéresse la bande côtière Roscoff - Cap Fréhel, décrite plus finement que dans le modèle global (pas de temps horaire et pas d'espace de $2 \times 2 \mathrm{~km}^{2}$, voir figure 5 ). Nous ne détaillerons pas ces modèles décrits par ailleurs par Agoumi et al., (1982) et Riou (1989).

Étalonné grâce à une série chronologique décennale (1960-1970) de températures acquises en baie de 
Morlaix par I'IFREMER (ex. ISTPM), le modèle côtier a été vérifié sur des données collectées d'une part en 1985 à proximité immédiate du laboratoire biologique de Roscoff (Sournia et al., 1987), d'autre part en 1987 entre Roscoff et St Brieuc (campagnes EDF héliportées); la précision du calcul est meilleure que le degré Celsius.

Étalonné sur les données fournies entre 1968 et 1971 par des bateaux commerciaux et 6 bateaux-feux (voir figure 8 ), le modèle global est moins fiable dans le domaine côtier qui nous intéresse, d'une part du fait de l'absence de bateau-feu à proximité et d'une moins bonne précision des mesures des bateaux commerciaux, d'autre part en raison de la plus grosse taille des mailles qui empêche de donner des informations au voisinage des côtes.

Ainsi, on conseillera au lecteur intéressé par l'intervalle côtier Roscoff - Cap Frehel de considérer en priorité les statistiques données par le modèle côtier (chapitre 3 ), les résultats du modèle global - donnant une image plus grossière - servant avant tout à faire ressortir les différences spatiales entre Brest et Port-en-Bes$\sin$ (chapitre 2).

Les statistiques issues du modèle concernent la période de 26 ans comprise entre 1962 et 1987 , et les résultats sont exprimés en nombre moyen de jours par an pendant lesquels les seuils thermiques $17,18,19$ et $20^{\circ} \mathrm{C}$ sont dépassés; dans cette approche, on considère les valeurs journalières de température de la colonne d'eau: on fait donc au préala- ble, jour par jour, une moyenne des températures calculées à chaque pas de temps; la température journalière de la colonne d'eau est obtenue en faisant la moyenne des températures de surface et de fond; notons que l'écart vertical à la côte ne dépasse pas $1^{\circ} \mathrm{C}$ en moyenne annuelle avec le modèle global (les valeurs les plus élevées sont observées entre Brest et Roscoff) et $0,3^{\circ} \mathrm{C}$ avec le modèle côtier Roscoff - Cap Fréhel.

\section{2. - RÉSULTATS DU MODĖLE GLOBAL}

Les statistiques de dépassement des températures $17,18,19$ et $20^{\circ} \mathrm{C}$ sont présentées sur les figures 1 à 4 pour les mailles les plus côtières.

Ces résultats mettent bien en évidence les deux endroits les plus chauds de la côte en été : d'une part le golfe normano-breton - entre St Brieuc à l'ouest et la latitude de Jersey à l'Est - d'autre part la limite Est de notre domaine d'étude entre la pointe de Barfleur et Port en Bessin. En particulier, le seuil $18^{\circ} \mathrm{C}$ y est dépassé plus de 30 jours par an en moyenne, ce qui se produit moins d'un jour par an ailleurs.

La particularité de ces deux endroits est liée à l'existence de profondeurs plus faibles à proximité de la côte (pente du talus continental moins aiguë) qui expliquent que les masses d'eau ont une inertie thermique plus faible et par conséquent répondent plus rapidement aux variations atmosphériques. 


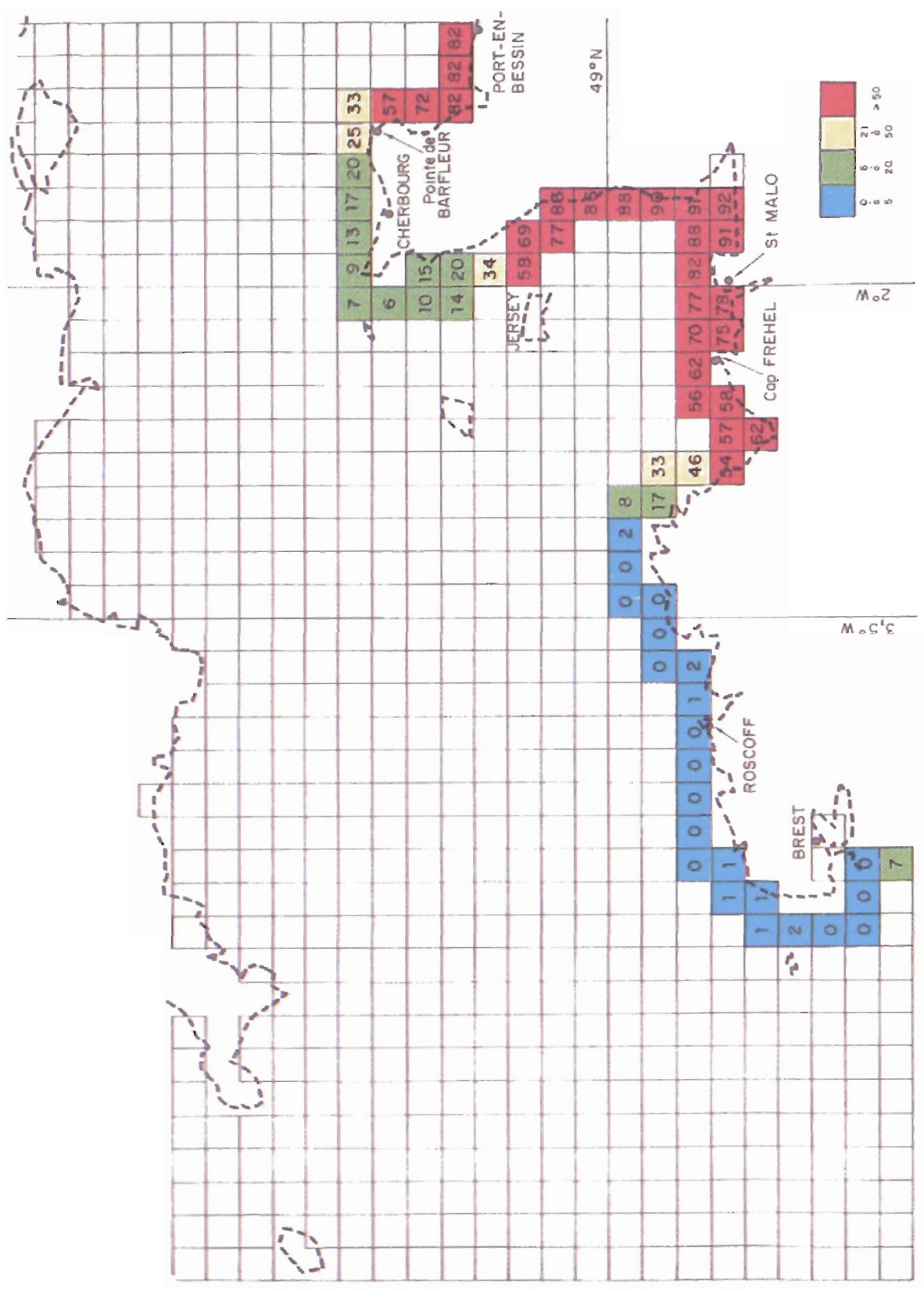

Fig. 1 - North Brittany and West Normandy coasts : number of days per year during which the mean temperature of the water column is greater than $17^{\circ} \mathrm{C}$; statistics for years $1962-1987$ from the global numerical model of the English Channel $\left(11 \times 11 \mathrm{~km}^{2}\right.$ mesh) 


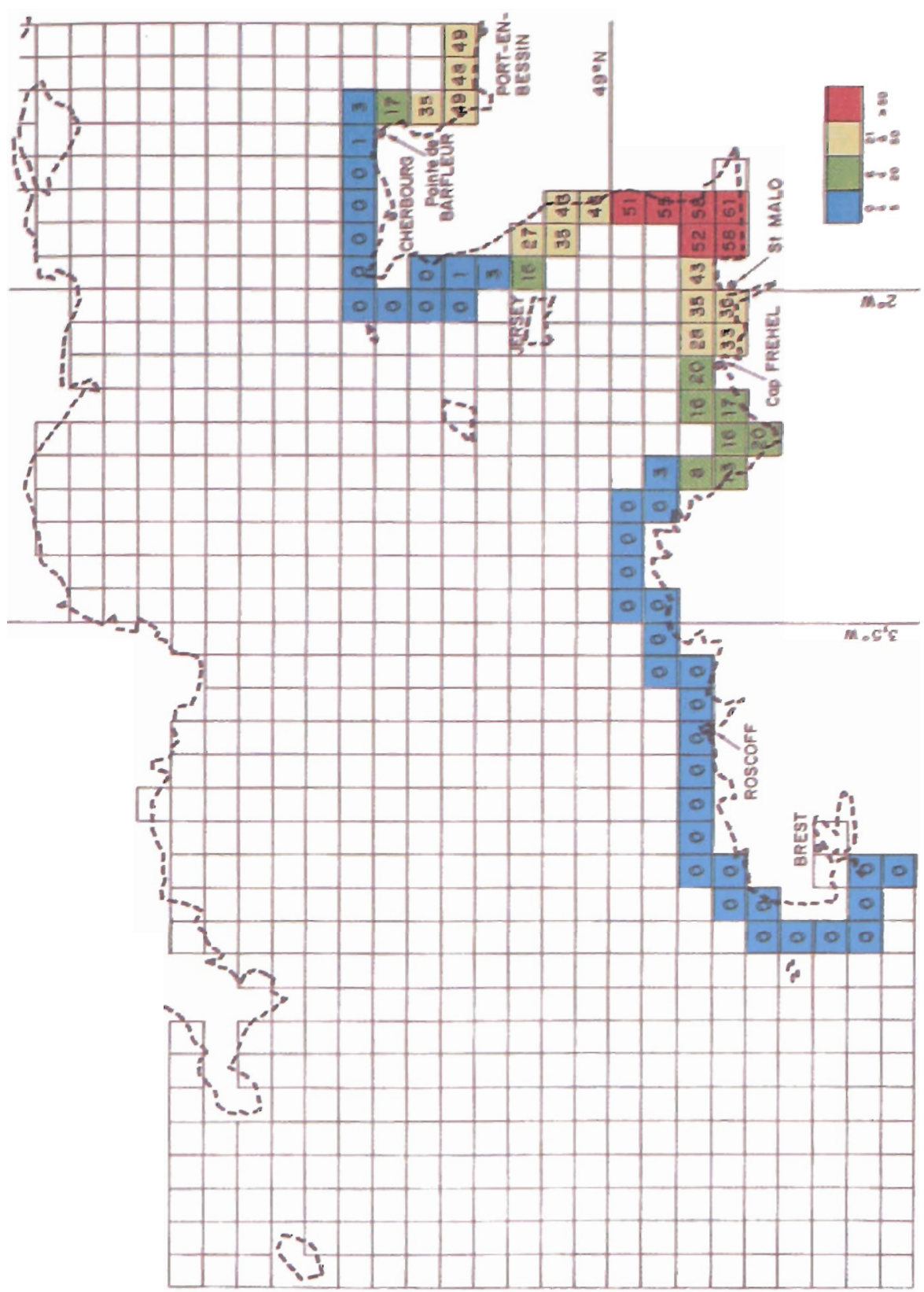

Fig. 2 - North Brittany and West Normandy coasts: number of days per year during which the mean temperature of the water column is greater than $18^{\circ} \mathrm{C}$; statistics for years $1962-1987$ from the global numerical model of the English Channel $\left(11 \times 11 \mathrm{~km}^{2}\right.$ mesh) 


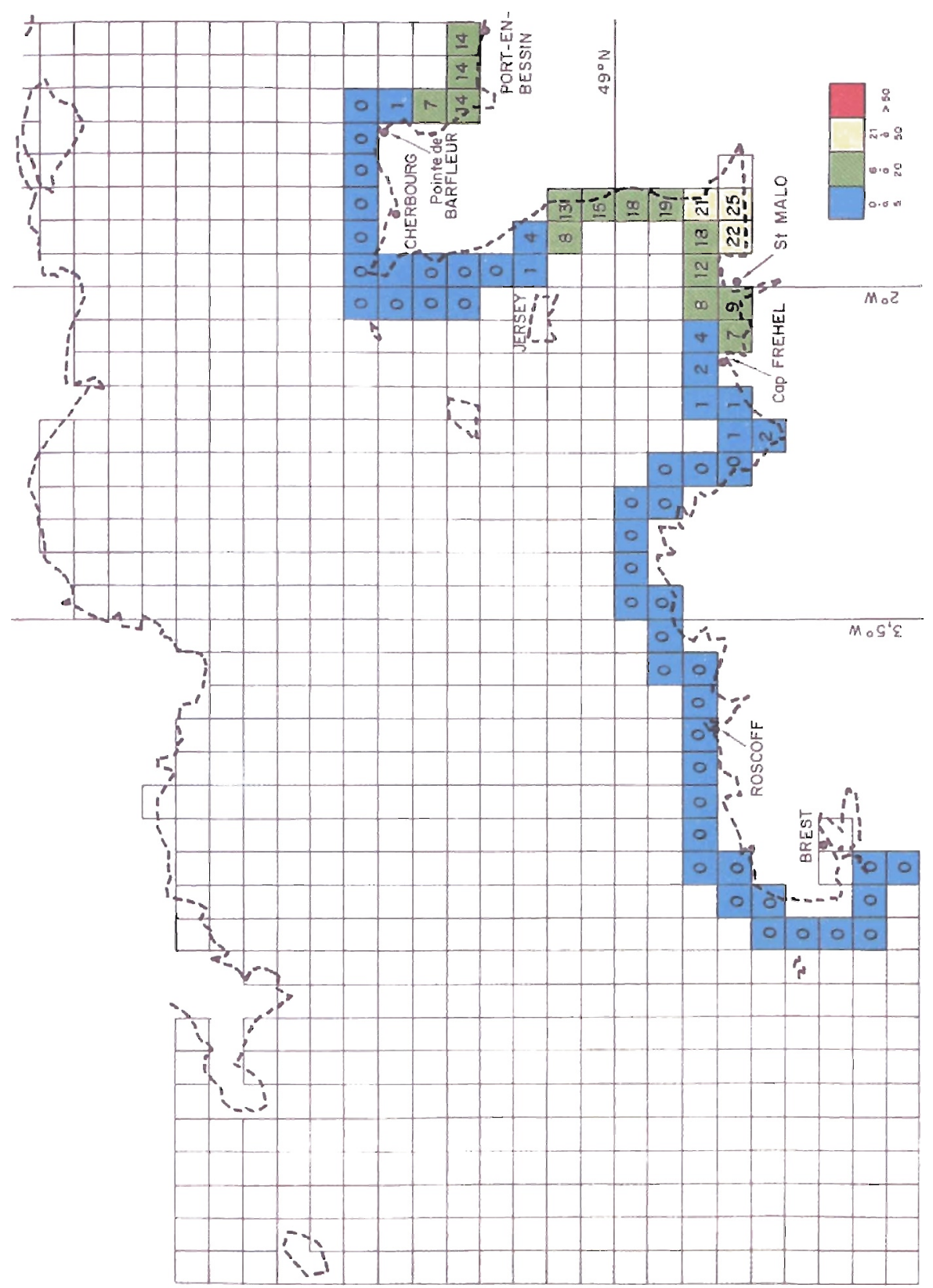

Fig. 3 - North Brittany and West Normandy coasts: number of days per year during which the mean temperature of the water column is greater than $19^{\circ} \mathrm{C}$; statistics for years $1962-1987$ from the global numerical model of the English Channel $\left(11 \times 11 \mathrm{~km}^{2}\right.$ mesh) 


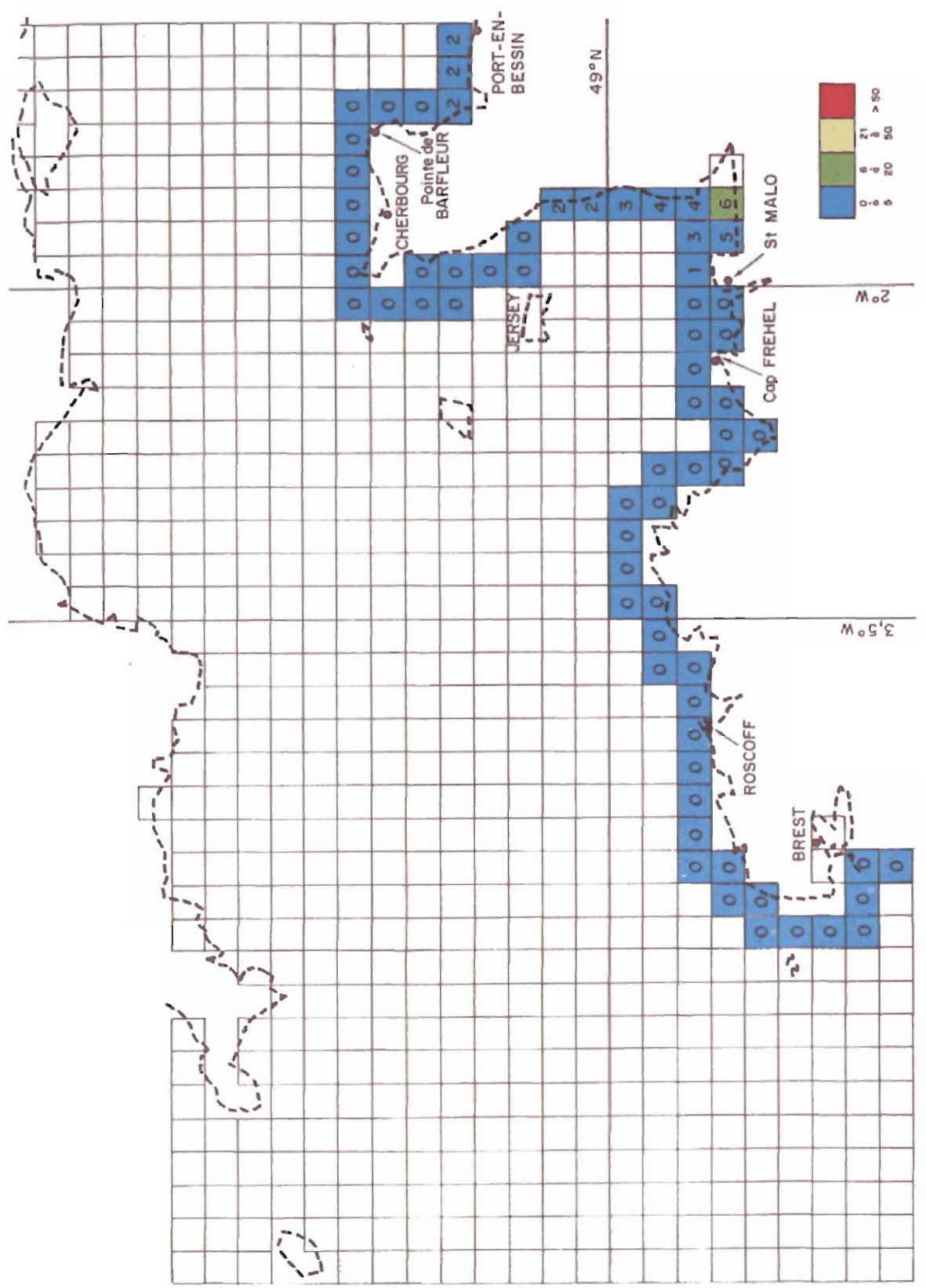

Fig. 4 - North Brittany and West Normandy coasts: number of days per year during which the mean temperature of the water column is greater than $20^{\circ} \mathrm{C}$; statistics for years 1962.1987 from the global numerical model of the English Channel $\left(11 \times 11 \mathrm{~km}^{2}\right.$ mesh) 


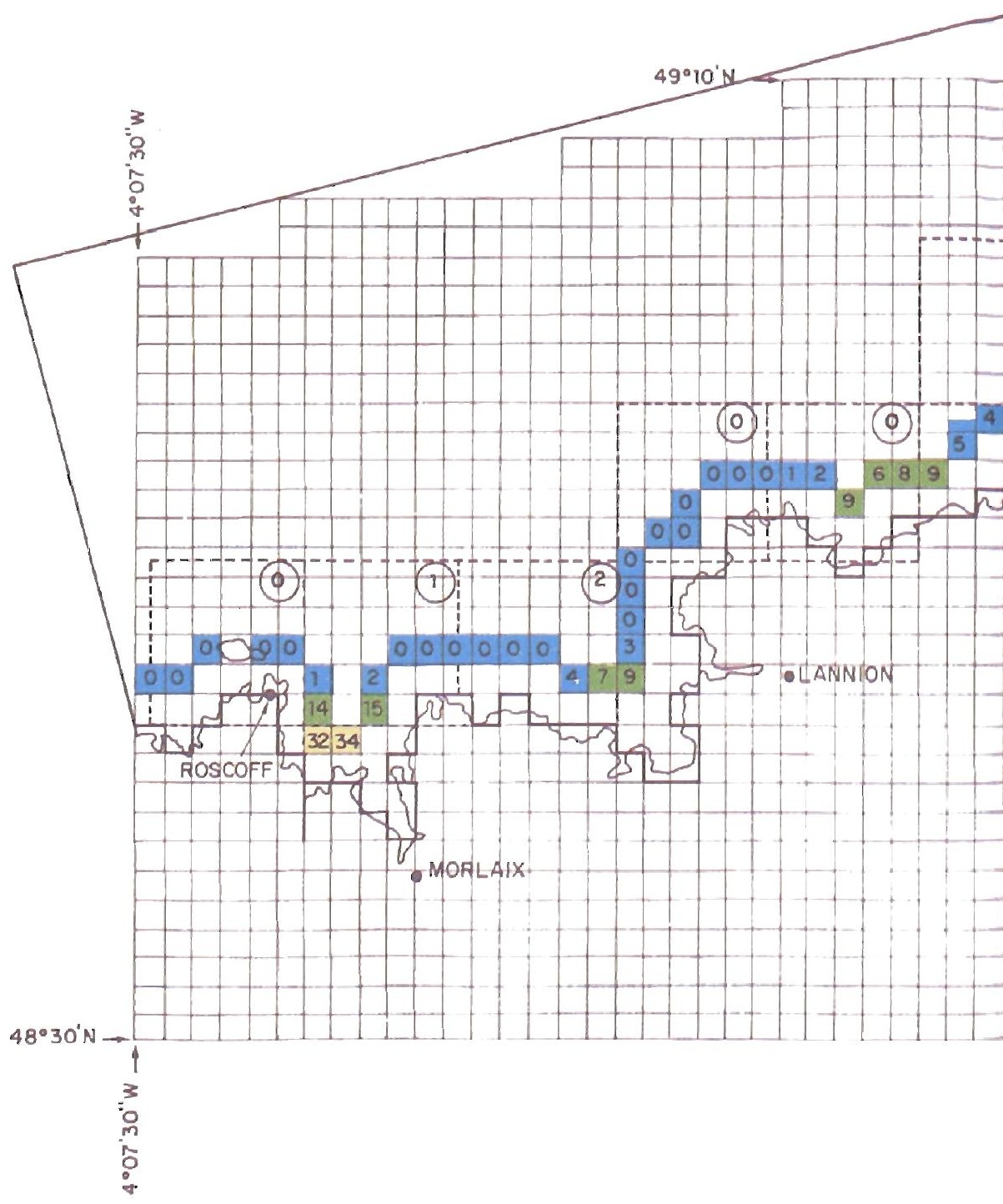

Fig. 5 - North-Brittany coast : number of days per year during which the mean temperature of the water column is greater than $17^{\circ} \mathrm{C}$; statistics for years $1962-1987$ resulting from either the global numerical model of the English Channel $\left(11 \times 11 \mathrm{~km}^{2}\right.$ dotted mesh, with results given in a circle placed in the upper right corner), and the coastal numerical model of North Brittany $\left(2 \times 2 \mathrm{~km}^{2}\right.$ mesh, with results given in the centre) 


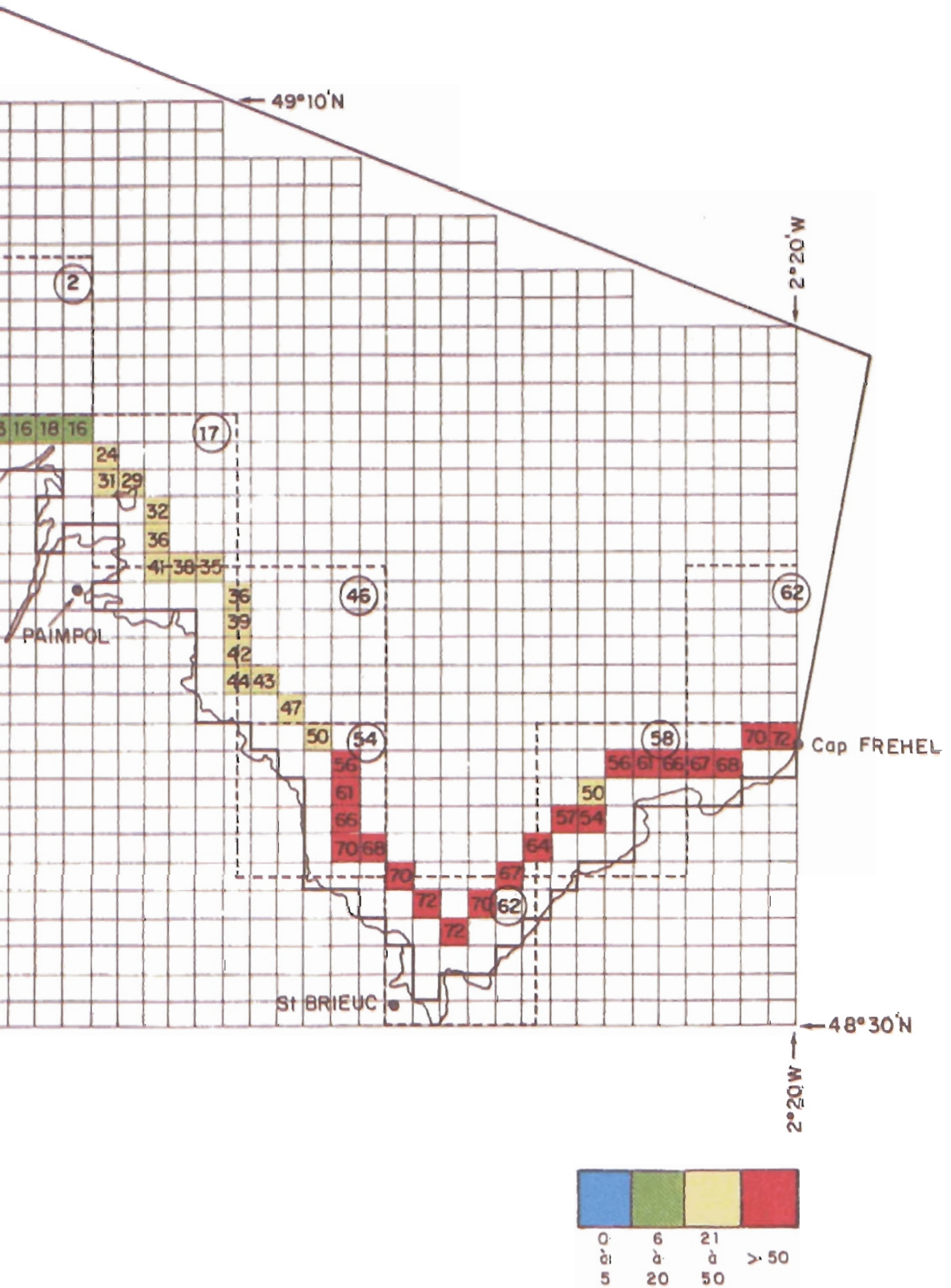

Fig. 5 - (suite) 


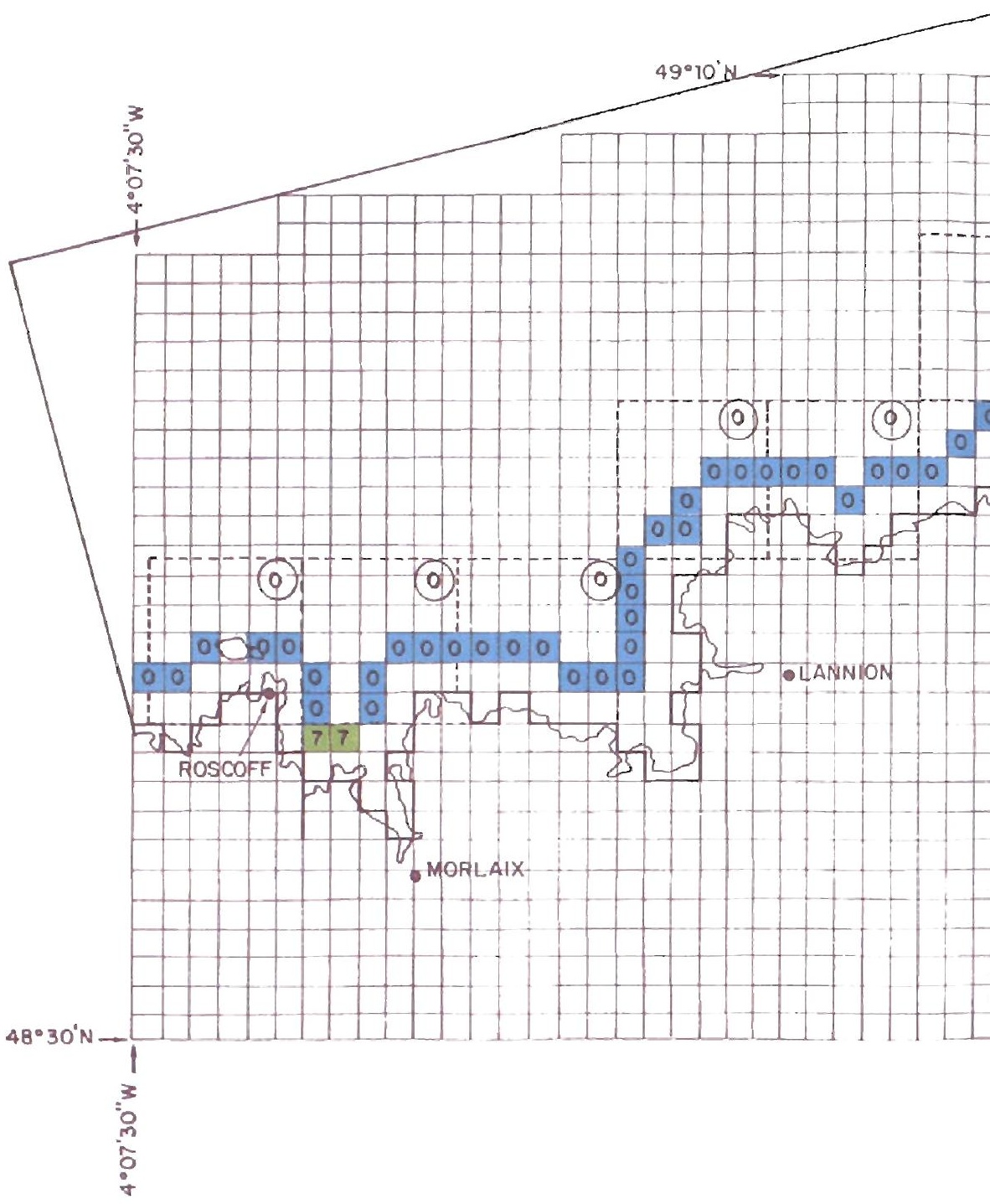

Fig. 6 - North-Brittany coast: number of days per year during which the mean temperature of the water column is greater than $18{ }^{\circ} \mathrm{C}$; statistics for years $1962-1987$ resulting from either the global numerical model of the English Channel $\left(11 \times 11 \mathrm{~km}^{2}\right.$ dotted mesh, with results given in a circle placed in the upper right corner), and the coastal numerical model of North Brittany $22 \times 2 \mathrm{~km}^{2}$ mesh, with results given in the centre) 


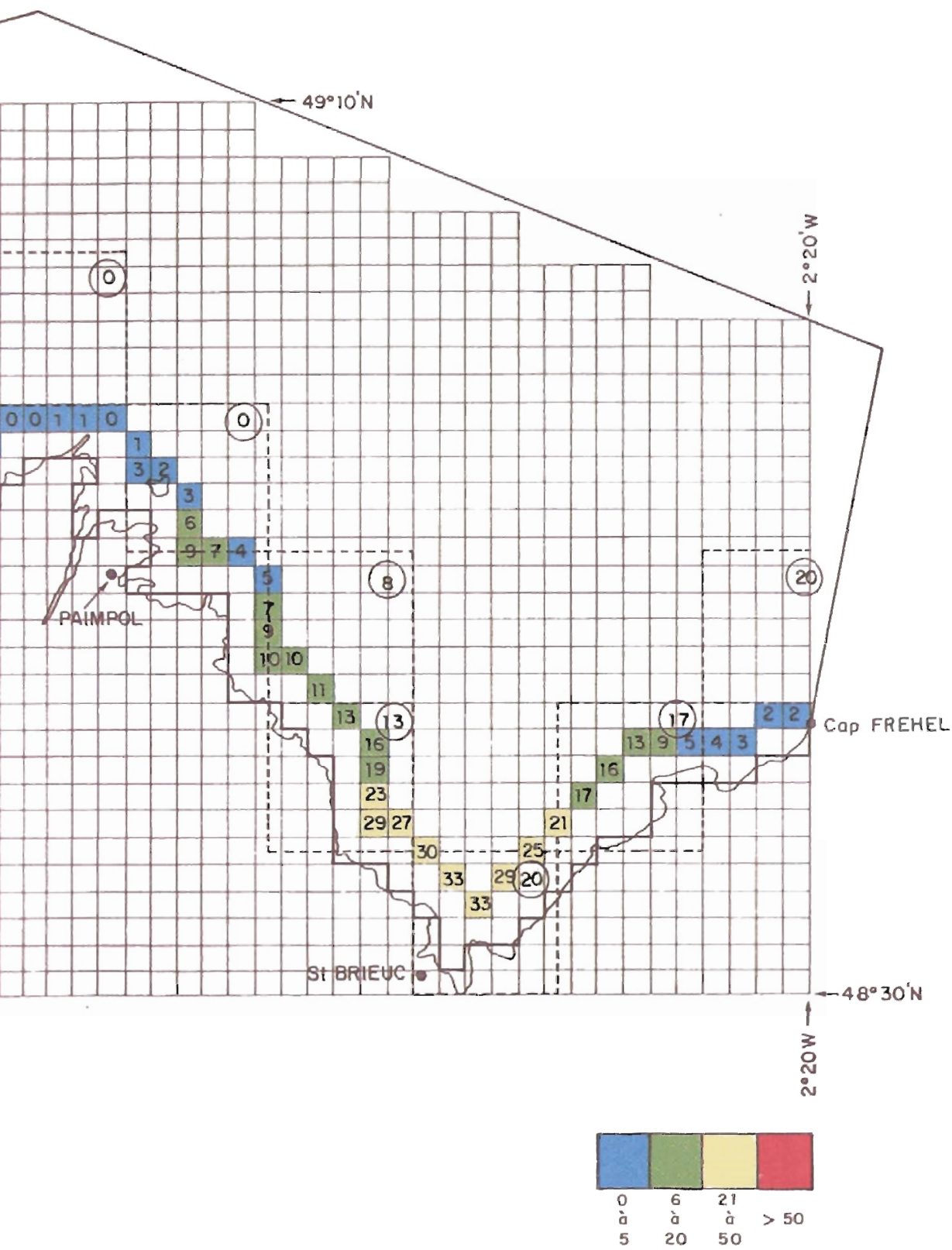

Fig. 6 (suite) 


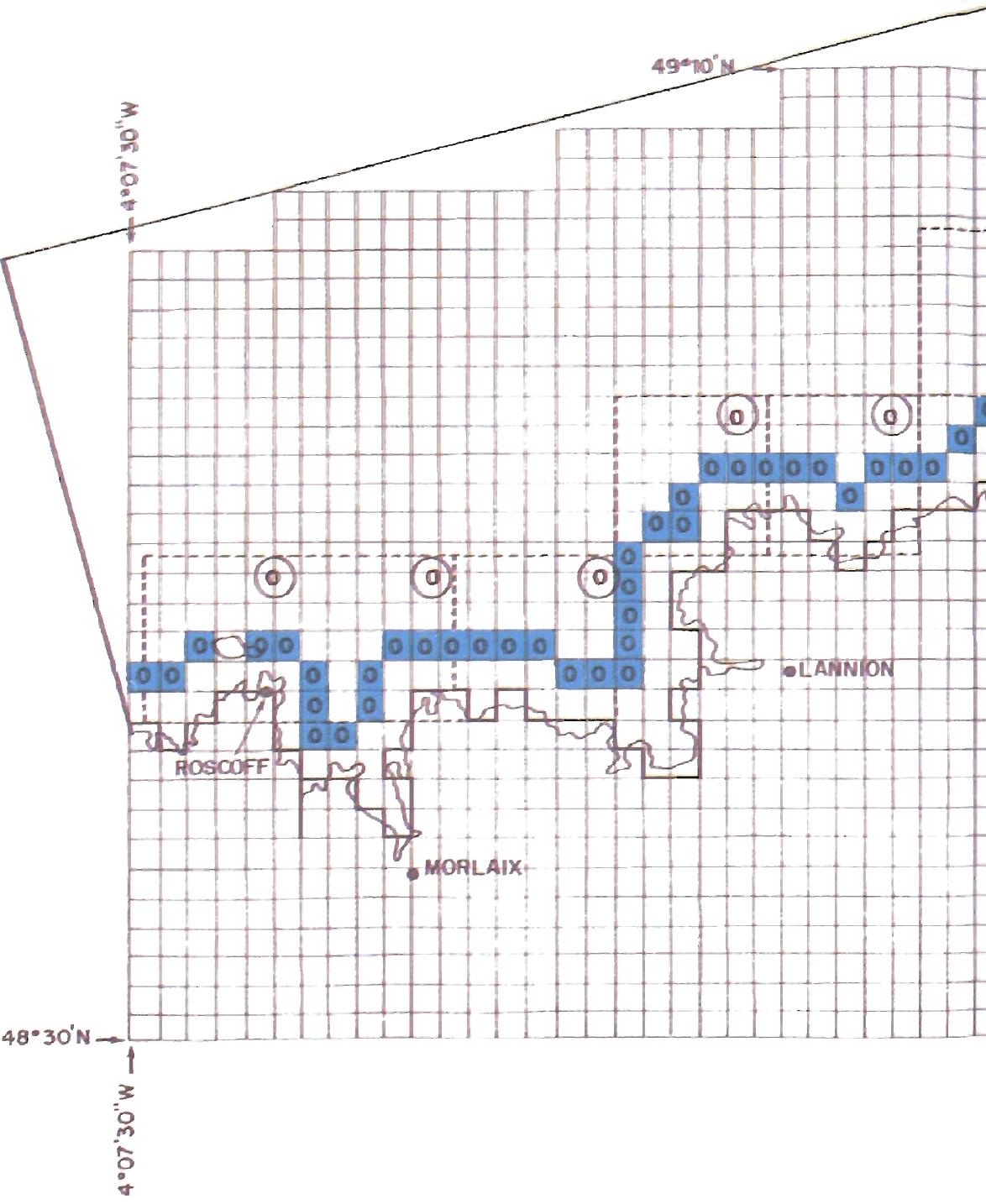

Fig. 7 - North-Brittany coast : number of days per year during which the mean temperature of the water column is greater than $19^{\circ} \mathrm{C}$; statistics for years $1962-1987$ resulting from either the global numerical model of the English Channel $\left(11 \times 11 \mathrm{~km}^{2}\right.$ dotted mesh, with results given in a circle placed in the upper right corner), and the coastal numerical model of North Brittany $\left(2 \times 2 \mathrm{~km}^{2}\right.$ mesh, with results given in the centre) 


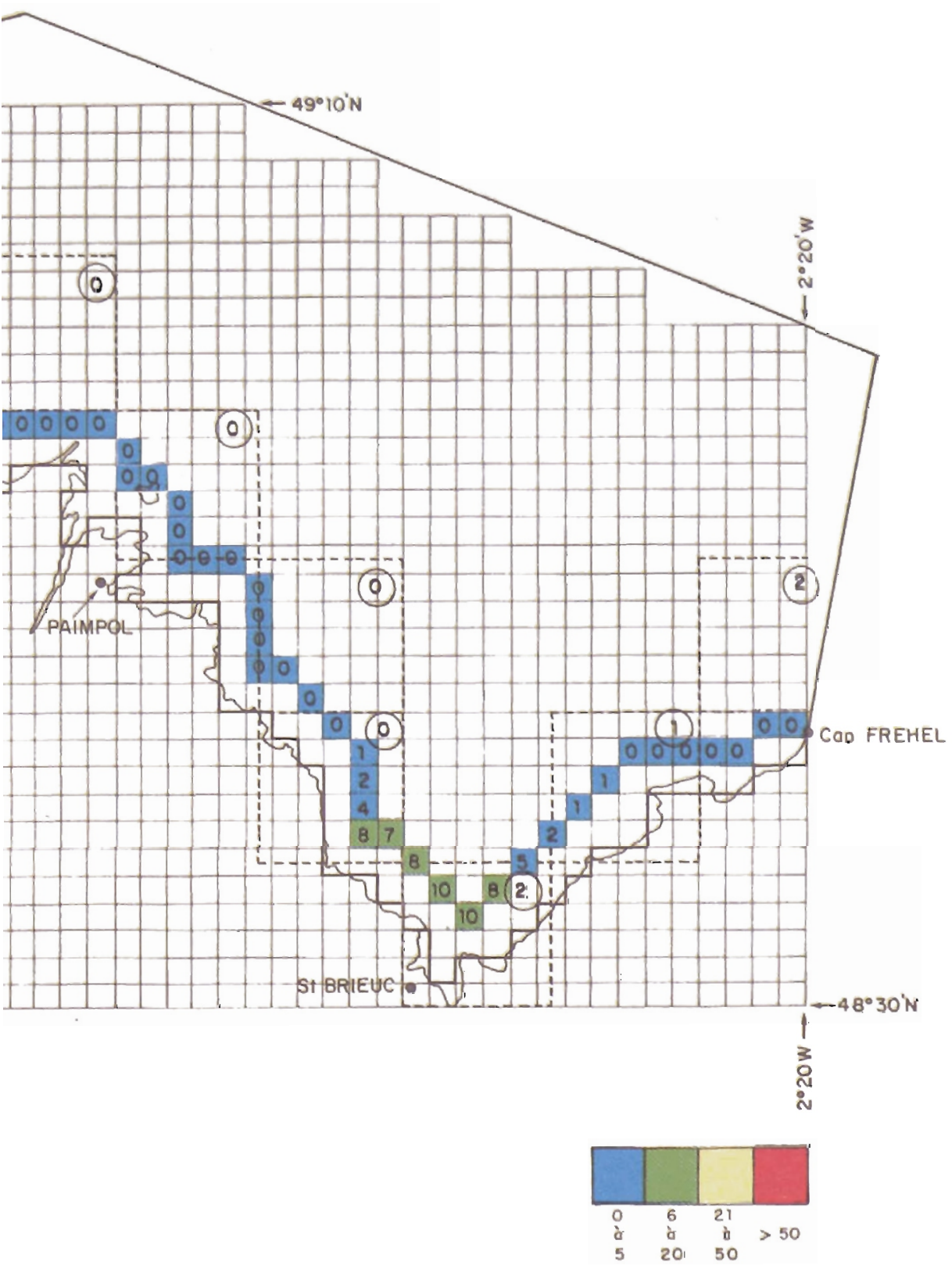

Fig. 7 (suite) 


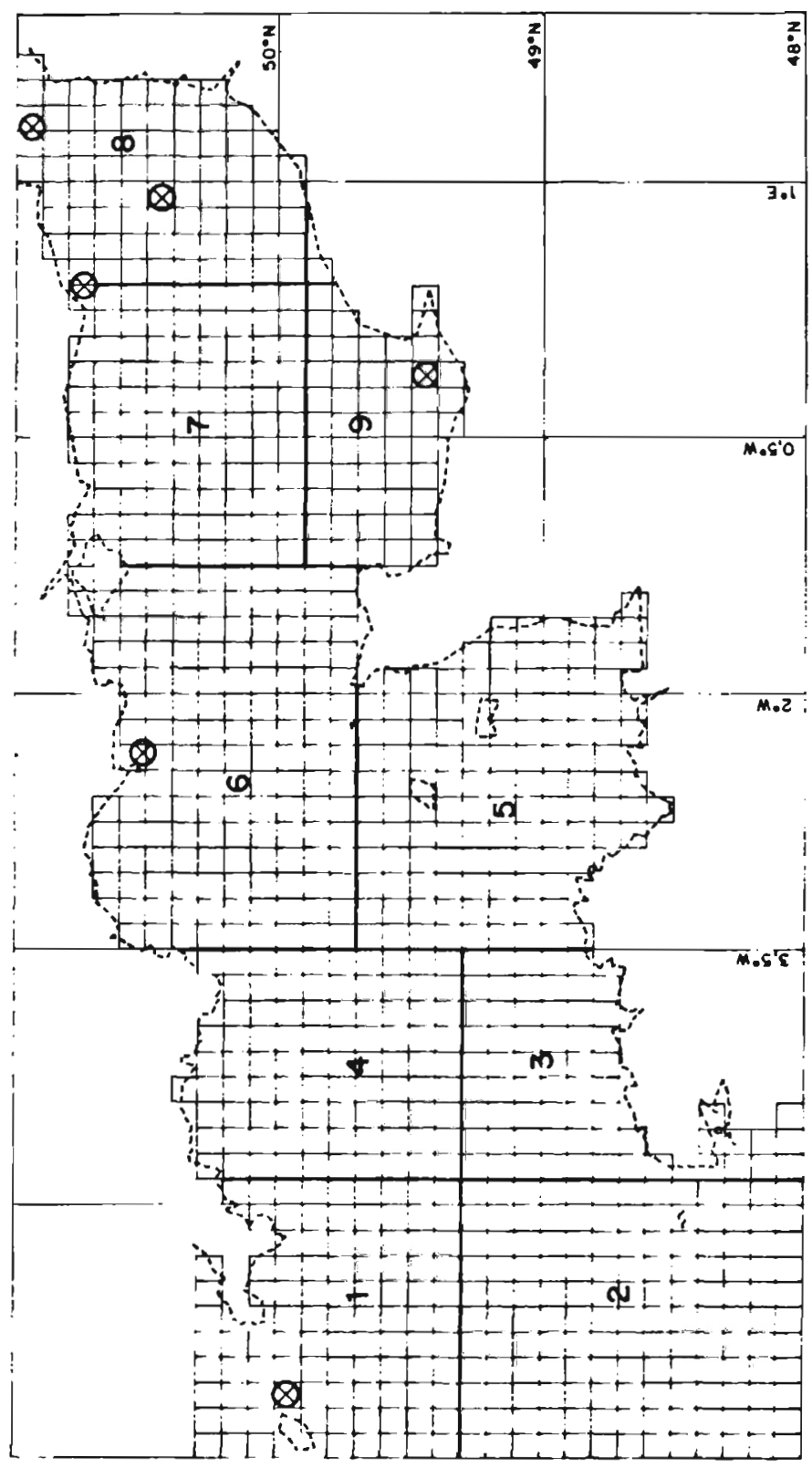

Fig. 8 - The area covered by the global numerical model of the English Channel $111 \times 11 \mathrm{~km}^{2}$ mesh) and position of the 6 light-ships used for the calibration of the model (1968-1971 period). Partition of the area into 9 sub-zones in which calibrated parameters (wind coefficient and dispersion coelficients $\mathrm{Kx}$ and $\mathrm{Ky}$ representing tides action) are homogeneous. 
On notera que dans le domaine étudié, la température $20^{\circ} \mathrm{C}$ n'est dépassée nulle part plus d'une semaine par an en moyenne sur les 26 ans.

\section{3. - RÉSULTATS DU MODĖLE COTIER}

Les statistiques de dépassement des températures 17,18 et $19^{\circ} \mathrm{C}$ établies à partir du modèle côtier sont présentées sur les figures 5,6 et 7 à une distance de l'ordre de $2 \mathrm{~km}$ à la côte pour s'affranchir des échauffements locaux qui pourraient survenir à quelques endroits et qui ne sont pas pris en compte (échauffement de la centrale de Flamanville, petits fleuves côtiers).

Par la finesse du maillage et la diversité des données thermiques utilisées pour son étalonnage et sa vérification, le modèle peut être considéré comme assez fiable. On a estimé que la précision des températures journalières calculées était supérieure à $1^{\circ} \mathrm{C}$ (Riou, 1989).

Le modèle côtier met également bien en évidence le domaine chaud en été du golfe normano-breton qui se distingue bien du secteur ouest aux eaux plus froides car plus profondes et donc d'inertie thermique plus grande. II faut noter que cette différence d'inertie joue dans le sens inverse en hiver avec des eaux plus froides à l'Est qu'à l'Ouest (Riou, 1989).

On a également indiqué sur les figures 5 à 7 les résultats donnés par le modèle global dans les mailles qui couvrent la bande côtière Roscoff Cap Fréhel.

Les résultats du modèle côtier et leur comparaison avec ceux du modèle global suscitent plusieurs remarques: tout d'abord, le fait de passer d'une grosse maille de $11 \times 11 \mathrm{~km}^{2}$ à une petite maille de $2 \times 2 \mathrm{~km}^{2}$ améliore très certainement la description des évolutions thermiques à la côte puisque l'on note des différences parfois sensibles entre deux petites mailles adjacentes; d'autre part les différences non négligeables qui apparaissent entre les résultats de certaines grosses mailles et les petites mailles qu'elles renferment montrent la limite de représentativité du modèle global soit en raison d'une taille trop grosse des mailles, soit d'un calage moins performant de ce dernier (voir remarques au chapitre 1).

\section{4. - CONCLUSION}

L'examen des figures 1 à 4 , qui présentent les statistiques de dépassement des températures $17,18,19$ et $20^{\circ} \mathrm{C}$ obtenues avec le modèle thermique global de la Manche, permet d'avoir une idée rapide des maxima thermiques observables à un endroit donné des côtes Nord Bretagne et Ouest Normandie.

Les figures 5 à 7 donnent une image plus fine et plus précise des températures élevées à la côte entre Roscoff et le cap Fréhel, le modèle côtier utilisé ayant une maillage 
$\left(2 \times 2 \mathrm{~km}^{2}\right)$ et une précision des calculs journaliers (meilleure que $1^{\circ} \mathrm{C}$ ) bien adaptés à l'étude de la côte.

Pour affiner les résultats donnés hors du champ d'étude du modèle côtier, on pourrait, dans un premier temps, - avant d'envisager le développement d'autres modèles côtiers améliorer la réponse du modèle global, en ajustant mieux les coefficients de ce modèle (en particulier les coefficients de diffusivité $\mathrm{Kx}$ et $\mathrm{Ky}$ qui traduisent l'effet dispersif des courants de marée sur les axes Ouest-Est et Nord-Sud) dans les zones 3 et 5 qui couvrent notre domaine d'étude (voir figure 8): on pourrait utiliser pour cette phase de calage les résultats du modèle thermique côtier déjà développé.

\section{BIBI_IOGRAPHIE}

Agoumi A., Enderle M.J., Gras R.A., 1982: Modélisation du régime thermique de la Manche. Oceanologica Acta, vol. $6, n^{\circ} 4$.

Enderle M., 1980 : Détermination du profil vertical de température d'une retenue de barrage : application au réservoir de Grangent sur la Loire. Électricité de France. Rapport EDF/DER/HE31/80, $n^{0} 33$.
Gras R.A., 1969 : Simulation du comportement thermique d'une rivière à partir des données fournies par un réseau classique d'observations météorologiques. IAHR Congress, Kyoto, 1969. Paper A53, Vol 1, p. 491-502.

Gras R.A., Gilbert A., Roult D., 1986 : Numerical computation of natural river temperatures. In Proceedings of International Conference on Water Quality modelling in Natural Environment. Bournemouth, 10-13 June 1986, pp. 457-472.

Klein P., et Bonnaud E., 1977 : Détermination du profil vertical d'une masse d'eau à partir des données météorologiques. Électricité de France. Rapport EDF/DER/HE31/77, $n^{\circ} 19$.

Klein P., 1980: Modélisation des mécanismes turbulents dans les couches marines superficielles (couche mélangée et thermocline). Thèse de Docteurès-Science. Université Aix-Marseille II.

Poulin M., 1980 : Modélisation du comportement thermique des cours d'eau. Application au Rhin. Thèse docteur-ingénieur. Université Paris VI.

Riou J., 1989: Modélisation du régime thermique sur le littoral nord-breton. Hydroécologie Appliquée, Vols 1/2, 1931.

Salençon M.J., 1987: Modélisation de l'eutrophisation des lacs et retenues. Eau et Développements. Revue marocaine de l'eau. 1987, n 4, pp. 68-84.

Sournia A., Birrien J.L., Douville J.L., Klein B., et Violler M., 1987: A daily study of diatom spring bloom at Roscoff (France) in 1985: I the spring bloom with the annual cycle. Estuarine, coastal and shelf science 25, 355-367. 